\title{
Lower Limb Differences and the Center of Pressure Control Test with Visual Feedback for Junior Soccer Players
}

\author{
Haruka Kawabata ${ }^{1, ~ *}$, Takaaki Miyake ${ }^{1}$, Izumi Yoshi ${ }^{1}$, Yutaro Kudo ${ }^{2}$ \\ ${ }^{1}$ Faculty of Liberal Arts and Science, Osaka Prefecture University, Sakai, Osaka \\ ${ }^{2}$ Undergraduate Department of Human Health, Hachinohe Gakuin University, Hachinohe, Japan
}

\section{Email address:}

kawabata@las.osakafu-u.ac.jp (H. Kawabata), tmiyake@las.osakafu-u.ac.jp (T. Miyake), yoshii@las.osakafu-u.ac.jp (I. Yoshii), kudo1026y@hachinohe-u.ac.jp (Y. Kudo)

${ }^{*}$ Corresponding author

\section{To cite this article:}

Haruka Kawabata, Takaaki Miyake, Izumi Yoshi, Yutaro Kudo. Lower Limb Differences and the Center of Pressure Control Test with Visual Feedback for Junior Soccer Players. American Journal of Sports Science. Vol. 9, No. 1, 2021, pp. 27-31. doi: 10.11648/j.ajss.20210901.14

Received: March 17, 2021; Accepted: April 2, 2021; Published: April 16, 2021

\begin{abstract}
This study aimed to compare the COP (center of foot pressure) control ability of junior soccer players between the kicking foot and the supporting foot using the COP control test. The participants were 40 junior soccer players $(133.6 \pm 5.4 \mathrm{~cm}$, $28.8 \pm 3.4 \mathrm{~kg}, 9.1 \pm 0.6$ years old, soccer history: $3.8 \pm 1.3$ years) belonging to a local soccer team. The COP control test was carried out using the COP trajectory measuring instrument T.K.K5810 and COP adjustment software 1.1.0 (Takei Scientific Instruments Co., Ltd.) to examine the lower right and left lower limb differences in the COP control ability. Of the 40 participants, 35 who kicked the ball with their right foot according to the questionnaire took part in the COP control test. The target-tracking COP control test tracks the target that moves regularly on the monitor with the COP and measures the total error over time. Each participant randomly practiced one standing position on both feet and one standing position (kicking foot and supporting foot), took a 30-second break between trials, and then performed two trials. The evaluation variable was total COP errors made in $30 \mathrm{~s}$ with the moving target. Of the two trials, for each player, the data with the best record were included for analysis. The mean COP control test scores when standing on one leg were $602.8 \pm 163.2 \mathrm{~cm}$ for the kicking leg and $561.6 \pm 159.1 \mathrm{~cm}$ for the supporting leg. The COP control test score with both feet standing was $487.0 \pm 146.2 \mathrm{~cm}$. The scores of one-foot standing (kicking foot and supporting foot) were significantly higher than those for participants standing on both feet $(\mathrm{p}<0.05)$, and many participants had better scores for their supporting foot than their kicking foot. However, there was no significant difference in the mean value of the kicking foot and the supporting foot and of the symmetry index, the effect size was also small. The results indicate that the COP control ability of junior soccer players does not differ between their kicking foot and their supporting foot.
\end{abstract}

Keywords: Balance, COP, Junior Soccer Players, Laterality

\section{Introduction}

Human limbs appear to be symmetrical, but they also have lateral functionality, exemplified by our ability to stand on one foot, footfall patterns in walking, and the ease with which we can stand on one leg or kick one foot while standing on the ground. There are also individual differences in functional laterality. For example, when using my lower limbs, I place much importance on the movements of each side and my "footedness" generally. The ability to identify which side one favors, is more stable, and is more skillfully dominant is important in sports and sports training. We used the body tracking test developed by Yoshida et al. [1,2] as a reference to develop a COP (Center of foot pressure) control test. Thereafter, we tested the test's reliability, sex differences, and the effects of aging on body balance control. We also sought to compare the results of the body balance control test and dynamic balance test $[3,4]$.

The characteristic of the COP control test utilizes "COP," a COP position convenient for measuring COP control ability and errors when a participant tracks a regularly moving target on a monitor. Therefore, the COP control test is evaluated for both feedback control and feedforward control. Previous studies of body balance control ability with participants of varying ages reported that laterality was not found in the 
lower limbs when participants were on the unstable board [5], they also did not observe laterality of muscular strength $[6,7]$. of the hip joint and knee joint. It is assumed that the supporting foot is more dominant than that of the kicking foot because the COP control test requires the ability to move the COP while maintaining a stable posture. In soccer, the supporting leg is opposite to the leg used for kicking the ball. In particular, we considered that junior soccer players who are still developing physically and acquiring skills tend to have more laterality; we thought that kicking and supporting legs were more independent than for senior soccer players.

We hypothesized that junior soccer players have laterality in their COP control ability.

The purpose of this study was to use the COP control test to examine the laterality of lower limbs in junior soccer players while maintaining the standing posture.

\section{Methods}

\subsection{Participants}

The participants were 40 boys (height: $133.6 \pm 5.4 \mathrm{~cm}$, weight: $28.8 \pm 3.4 \mathrm{~kg}$, age: $9.1 \pm 0.6$ years old, soccer carrier: $3.8 \pm 1.3$ years) among the junior soccer players who belonged to the local soccer team. All participants provided their consent to participate in the study. None of the participants had any injuries to the lower limbs or wore glasses or contact lenses. Prior to conducting this study, we sufficiently explained the purpose of the experiment to participants and coaches, as well as the procedure and that the study did not involve any invasive techniques or measurements for the body.

This research was conducted with the approval of the Research Ethics Committee of Osaka Prefecture University.

\subsection{Laboratory Instrument}

The laboratory equipment used was a centroid oscillation system T.K.K5810 (Takei Scientific Instruments Co., Ltd. Japan) with four built-in acceleration sensors for Windows 10 . The device was connected to a Windows PC and a 27-inch monitor (resolution: $1920 \times 1080$ ). The body balance control test conducted with these systems measures errors between the moving target on the monitor and the participant's COP over time and was developed based on previous studies

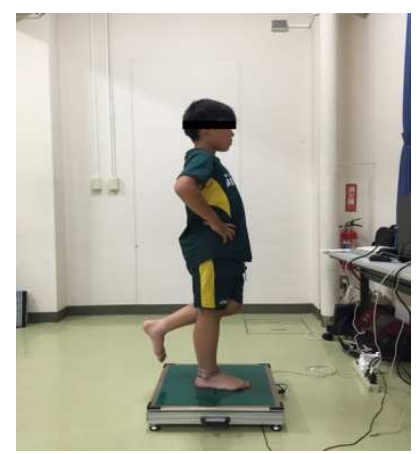

Figure 1. Posture of the participate during the COP control test.

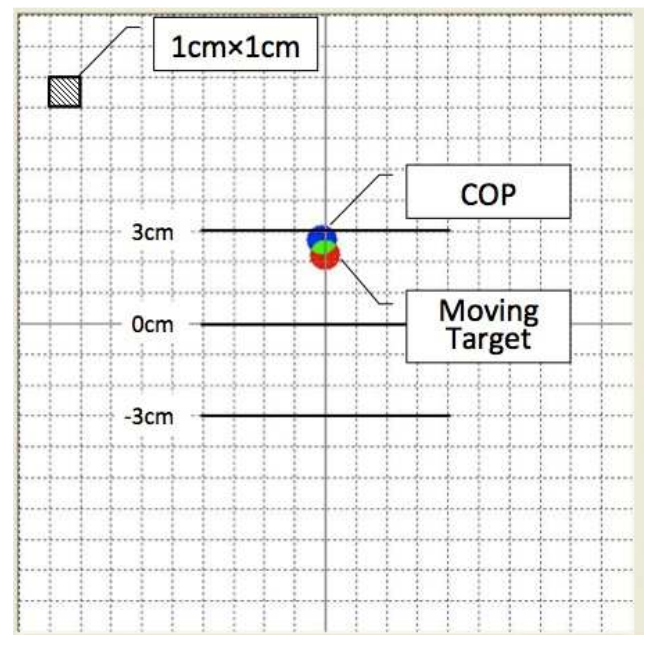

Figure 2. Screen display.

\subsubsection{Experimental Procedure}

A questionnaire survey was conducted with participants to investigate the lower limbs for selection for the exercise task. Demura et al. [8] reported that it was possible to determine the dominant leg using only four question items about four activities (hopping, putting a foot on a table, kicking the ball, stomping objects) with the Waterloo Footedness Questionnaire-Revised [9]. Therefore, the survey questions used were about the following: foot used for bouncing or hopping, putting feet on a table, foot used to kick the ball, and foot used to stomp objects. The participants answered using the two-case method (right foot or left foot) (Table 1).

Table 1. Four question items about four activities.

\begin{tabular}{llll}
\hline 1. hopping & Right & $\cdot$ & Left \\
2. putting a foot on a table & Right & $\cdot$ & Left \\
3. kicking the ball & Right & $\cdot$ & Left \\
4. stomping objects & Right & $\cdot$ & Left \\
\hline
\end{tabular}

\subsubsection{The COP Control Test}

The sampling time was $30 \mathrm{~s}$; the sampling frequency was $50 \mathrm{~Hz}$; the size of the moving target and COP target was 1 $\mathrm{cm}$ in diameter; the speed of the moving target was $1 \mathrm{~cm} / \mathrm{s}$; and the grid displayed on the monitor was $1 \mathrm{~cm}^{2}(1 \mathrm{~cm} \times 1$ $\mathrm{cm})$. The moving width of the moving target was set to $3 \mathrm{~cm}$ forward and backward from the center of the coordinates on the Y-axis before the measurement, the examiner explained that the participants should not move their foot when following the moving target, but should shift their weight, keeping their feet in the same place. If the target moved upward (forward), the participant should move the COP forward, pressing through the front of the foot (toe direction). When the target moved toward the participant (backward), the participant was instructed to move the COP backward (heel direction), pressing through the back part of the foot.

The participants stood on one or both feet, with both hands on their hips, on a grounded measuring instrument on a horizontal floor. The width between the inside of one foot to the inside of the other foot when standing on both legs was $10 \mathrm{~cm}$. We began taking measurements after confirming that 
the participant's posture was stable. The COP of each participant was recorded before the start of measurement so that the measurement could follow from the origin at the same time as the start of measurement (similar to adjusting for tare weight to get accurate net weight). The position of the monitor was $1.0 \mathrm{~m}$ away from the participant, and the moving target was placed so that the start position was at the participant's eye level. The error between the moving target and the COP following it was recorded for $30 \mathrm{~s}$ from the start of measurement.

The COP control test was performed standing on one foot (randomly chosen) and then standing on both feet. Participants had one practice trial time and two main trial times. The break between trials was $30 \mathrm{~s}$. The best score of the two trials was used as the representative value of the participants.

The test was performed after confirming the stability of the participant's posture. Any participant who lost their balance within the first ten seconds, leaving them on one foot, or separated a hand from their waist was tested again (none of the participants failed twice). The test score was the sum of the errors between the moving target and the COP in $30 \mathrm{~s}$; the smaller the score, the better the COP control ability.

The symmetry index (SI) [10] was calculated using the following formula to examine the degree of left-right difference in the lower limbs. In the present study, the dominant and non-dominant legs were not defined, so a good score was assigned to the dominant limb, and the score of the other step was assigned to the non-dominant limb.

\subsubsection{Statistical Analysis}

The results are shown as the mean \pm standard deviation and frequency (relative value). In order to analyze the questionnaire results after the independence test ( $\chi 2$ test), we performed Haberman residual analysis. Multiple comparison tests using unpaired one-factor analysis of variance and Bonferroni's method were performed to compare the mean values of the dynamic balance abilities of the right foot, left foot, and both feet. We used Hedge's g to calculate the effect amount indicating the magnitude of the difference between the average values and the Welch method to test the difference between the mean values of the two groups. The statistical significance level in this study was set at 5\%. SPSS Statistics version 25 (IBM) was used for statistical analysis.

\section{Results}

\subsection{Lower Extremities Selected for the Exercise Tasks}

Table 2 shows the results of the questionnaire survey answered by 40 participants. The total number of right-footed respondents was $118(73.7 \%)$; the number who were left-footed was $42(26.3 \%)$. The right foot was $23(57.5 \%)$, and the left foot was $17(42.5 \%)$. The right foot was 29 $(72.5 \%)$, and the left foot was $11(27.5 \%)$. The right foot was $35(87.5 \%)$, and the left foot was $5(12.5 \%)$. The right foot was $31(77.5 \%)$, and the left foot was $9(22.5 \%)$. Results of the $\chi^{2}$ test indicate a significant difference $(\chi 2=9.685, \mathrm{Df}=3)$ was found, multiple comparison test was carried out. Multiple comparison test showed that the right foot often kicked the ball, but did less hopping. On the other hand, the left foot often hopped, but did less hopping.

Table 2. Lower extremities selected for the exercise tasks and results of the $\chi^{2}$ test.

\begin{tabular}{llllll}
\hline & Right foot & Left foot & Total & $\chi \mathbf{2}$ & p \\
\hline Foot used for & 23 & 17 & 40 & & \\
bouncing or & $57.5 \%$ & $42.5 \%$ & $100 \%$ & & \\
hopping & $\left(-2.70^{*}\right)$ & $(2.70 *)$ & & & \\
Putting feet on & 29 & 11 & 40 & & \\
a table & $72.5 \%$ & $27.5 \%$ & $100 \%$ & & \\
& $(-0.21)$ & $(0.21)$ & & 9.69 & 0.02 \\
Foot used to & 35 & 5 & 40 & & \\
kick the ball & $87.5 \%$ & $12.5 \%$ & $100 \%$ & & \\
& $(2.28 *)$ & $(-2.28 *)$ & & & \\
Foot used to & 31 & 9 & 40 & & \\
stomp objects & $77.5 \%$ & $22.5 \%$ & $100 \%$ & & \\
Total & $(0.62)$ & $(-0.62)$ & & & \\
& 118 & 42 & & & \\
\hline
\end{tabular}

$※$ ( ): adjusted residual $*$ :p $<0.05$

\subsection{Comparison of the COP Control Test for Kicking Foot, Supporting Foot, and Standing on Both Feet}

Table 3 shows the average and standard deviation of the COP control test. The participants of the body balance control test were 35 people who answered that they kicked the ball with their right foot. The right foot was $602.8 \pm 163.2 \mathrm{~cm}$, the left foot was $561.6 \pm 159.1 \mathrm{~cm}$, and both feet were $487.0 \pm 146.2$ $\mathrm{cm}$. As a result of paired one-way analysis of variance, a significant difference was found between the groups $\left(\mathrm{F}_{(2)}=\right.$ 4.938). As a result of multiple comparison tests, no significant difference was found between the right and left feet, and both feet were significantly larger than the right and left feet. The effect sizes were 0.26 for the right and left feet, 0.75 for the right and both feet, and 0.49 for the left and both feet.

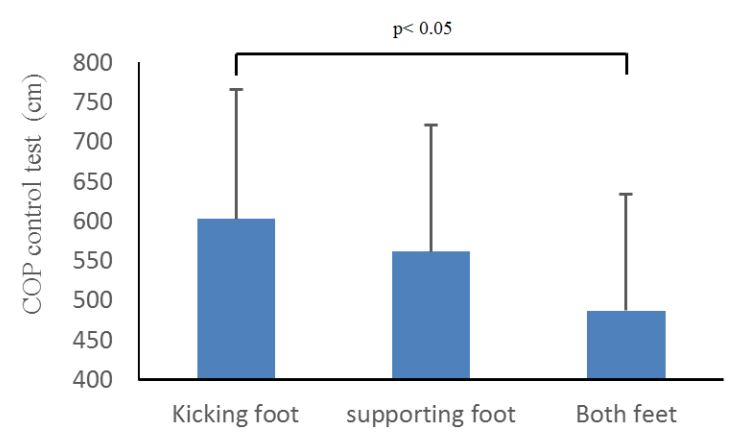

Figure 3. Comparison of the COP control test for kicking foot, supporting foot, and standing on both feet.
※ Effect size (Hedges')
1. Kicking foot - supporting foot: 0.55
2. Kicking foot - both feet: 0.75
3. supporting foot - both feet: 0.49

Table 3 shows the individual performance and symmetry index (SI) when standing on one foot on the left and right by the COP test. The kicking foot was superior to the supporting 
foot in $14(40.0 \%)$, and the supporting foot was superior to the kicking foot in $21(60.0 \%)$. The SI calculated as an index showing the left-right difference of the lower limbs was $10.7 \pm 7.5$ in the group with good right leg performance and
$15.3 \pm 13.2$ in the group with good left leg performance. No significant difference was found in the mean values of the two groups. The effect size was 0.43 .

Table 3. Comparison of the COP control test for right-footed and left-footed.

\begin{tabular}{llllll}
\hline & Right-footed 14 boys (40.0\%) & Left-footed 21 boys (60\%) & t & ES & p \\
\hline Symmetry index (SI) & $10.7 \pm 7.5$ & $15.3 \pm 13.2$ & 1.78 & 0.19 \\
\hline
\end{tabular}

※ ES: Effect Size ( Hedges'g )

\section{Discussion}

Based on the test of independence ( $\chi 2$ test), the relationship between the right foot and the kicking foot of the ball was confirmed, so it can be said that the kicking motion of the ball is more likely to found superior to other motions. For this reason, we analyzed 35 boys who used their right foot to kick.

Since the age of 35 participants was $9.1 \pm 0.6$ years old, their soccer skills are immature and their movements are biased, it is assumed that there is a left-right difference in the lower limbs for exercise tasks.

However, no significant left-right difference was found in the mean value and SI, and the effect size showing the magnitude of the mean value was also small. The average SI used as an index of left-right difference was 13.5 \pm 11.4 . Generally, values below 15 [11] are considered to be within the normal range of 1 . It was suggested that the difference between the right and left of the participants was not large, but the individual difference was large.

Grentry and Gabbard [12] judged the dominant foot of 956 males and females aged $4,8,11,13,16$, and 20 years, and the right foot in manipulating an object (a ball). They reported that $66 \%$ or more of the total was 4 to 8 years old and $81 \%$ after 11 years of age. Gabbard and Iteya [13] reported that children up to the age of 11 had a mixed foot phenomenon in which the lower limbs had less lateral difference. In any case, it is considered that lower extremity dominance becomes more prominent on the right side with developmental development.

In the COP control test, there was no difference in left and right limbs, but the ability to control the COP when standing on one foot was lower than that on both feet. The reason for this may be that it became difficult to maintain the posture with the decrease in the base of the support, and the increase in the load on the lower limbs caused muscle fatigue, increasing the amount of shaking.

As a result of calculating the ratio of each of the kicking foot and the supporting foot to the score when standing on both feet, the supporting foot $(561.6 \pm 159.1 \mathrm{~cm})$ increased $15.3 \%$ compared to both feet $(487.0 \pm 146.2 \mathrm{~cm})$, while the kicking foot $(602.8 \pm 163.2 \mathrm{~cm})$ increased by $23.8 \%$, which is 8.5 points higher than the supporting foot. However, there was no significant difference in the ratio between the two.

Coren [14] and Peters [15] classified the lower limbs as the legs, used for manipulating objects, kicking the ball, and maintaining posture. When kicking the ball, the ground reaction force on the supporting foot increases in proportion to the number of steps and speed of the approach run. Therefore, when transmitting a large force to the ball, the support foot must be highly stable. However, since bipedal walking is a daily activity, we also need stability in the kicking foot. If the right and left COP control abilities differ remarkably, it may cause an imbalance when engaging in physical activities.

When writing letters, holding chopsticks, operating a mobile phone, and so on, with the upper limbs, we tend to favor one of them, demonstrating the one-sided functional advantages [14-19]. However, the lower limbs play an important role in supporting weight, and the upper limbs do not. Additionally, since the lower limbs perform symmetrical movements such as standing, walking, and running, the difference between left and right is smaller than that of the upper limbs. The fact that there is no left-right difference in lower limb function may also be an advantage in that the fatigue and load on the lower limbs is dispersed, and movements can be performed smoothly with either the left or right foot. Furthermore, the lower limbs of humans have some functional or sensory left-right differences in controlling the COP, but the differences are not large and are considered to occur within a certain range.

In the field of health and sports science, there have been many reports on the difference between the left and right limbs. When humans learn the technique of utilizing left-right asymmetry and the technique improves, a functional left-right difference appears regardless of sex. On the other hand, with the improvement of technology, the left-right difference becomes smaller, and in some cases, the performance can be demonstrated equally on the left and right.

In the present study, there were few differences in the left and right limbs in humans. However, it was found that it is difficult to examine the degree of left-right difference because the degree of appearance varies depending on the individual. We would like to continue researching sports skills and functional differences, to provide useful information to people who play sports.

\section{Conclusion}

This study aimed to compare the COP control ability of junior soccer players between the kicking foot and the supporting foot using the COP control test. The participants were 40 junior soccer players belonging to a local soccer team. Of the 40 participants, 35 who kicked the ball with their right foot according to the questionnaire took part in the COP 
control test. Many participants had better scores for their supporting foot than their kicking foot. However, there was no significant difference in the mean value of the kicking foot and the supporting foot and of the symmetry index, the effect size was also small. From the above, the COP control ability of junior soccer players does not differ between their kicking foot and their supporting foot.

\section{Acknowledgements}

This research was supported by JSPS Grants-in-Aid for Scientific Research 26870225, and we would like to express our gratitude to them.

\section{References}

[1] Yoshida T, Oda M, Takahashi H, Sasaki S, Yamamoto M, Takenouchi J. (1996). The Relationship for Target and Tracking of the Body Tracking Test (BTT) The Test Condition for the BTT-No.1. Equilib Res. 55, 4, 343-348.

[2] Yoshida T, Oda M, Osafune H, Miyaji M, Yamamoto M. (1997) The Evaluation of Tracking Ability by the Body Tracking Test (BTT). Equilib Res. 56, 1, 39-44.

[3] Kawabata H, Uchiyama M, Demura S, Takahashi K. (2013). Relations among dynamic balance tests and a coordination test using center of pressure to pursue a randomly moving target. Percept Mot Skills. 117, 3, 811-820.

[4] Kawabata H, Demura S, Uchiyama M. (2012). Reliability and Sex Differences in a Coordination Test of a Tracking moving target with the Center of Foot Pressure. Adv Phys Educ, 02, 03, $77-81$

[5] Huurnink A, Fransz DP, Kingma I, Hupperets MDW, Van Dieën JH. (2014). The effect of leg preference on postural stability in healthy athletes. J Biomech, 47, 1, 308-312.

[6] Burnie J, Brodie DA. Isokinetic measurement in preadolescent males. (1986). Int J Sports Med, 07, 4, 205-209.

[7] Neumann DA, Soderberg GL, Cook TM. (1988). Comparison of maximal isometric hip abductor muscle torques between hip sides. Phys Ther, 68, 4, 496-502.
[8] Demura S, Sato S, Sugiura H. (2010). Lower limb laterality characteristics based on the relationship between activities and individual laterality. Gazz Medica Ital Arch per le Sci Mediche, 169, 5, 181-191.

[9] Elias LJ, Bryden MP, Bulman-Fleming MB. (1998). Footedness is a better predictor than is handedness of emotional lateralization. Neuropsychologia, 36, 1, 37-43.

[10] Schiltz M, Lehance C, Maquet D, Bury T, Crielaard JM, Croisier JL. (2009). Explosive strength imbalances in professional basketball players. J Athl Train, 44, 1, 39-47.

[11] Noyes FR, Barber SD, Mooar LA. (1989). A rationale for assessing sports activity levels and limitations in knee disorders. Clin Orthop Relat Res, 246, 238-249.

[12] Gentry V, Gabbard C. (1995). Foot-preference behavior: A developmental perspective. J Gen Psychol, 122, 1, 37-27.

[13] Gabbard C, Iteya M. (1996). Foot laterality in children, adolescents, and adults. Laterality, 1, 3, 199-206.

[14] Coren S. (1993). The lateral preference inventory for measurement of handedness, footedness, eyedness, and earedness: Norms for young adults. Bull Psychon Soc, 31, 1, 1-3.

[15] Peters M. (1988). Footedness: Asymmetries in Foot Preference and Skill and Neuropsychological Assessment of Foot Movement. Psychol Bull, 103, 2, 179-192.

[16] Kawabata H, Demura S, Kitabayashi T, Shin S, Sato S. (2012). Gender and the laterality of various coordination tests. Gazz Medica Ital Arch per le Sci Mediche, 171, 2, 135-141.

[17] Noguchi T, Demura S, Nagasawa Y, Uchiyama M. (2009). Influence of measurement order by dominant and nondominant hands on performance of a pursuit-rotor task. Percept Mot Skills, 108, 3, 905-914.

[18] Roy EA, Bryden P, Cavill S. (2003). Hand differences in pegboard performance through development. Brain Cogn, 53, 2, 315-317.

[19] Bell J, Gabbard C. (2005). Foot preference changes through adulthood. Laterality, 5, 1, 63-68. 